\title{
ON A SAFETY CRITERION FOR COLUMN-LIKE STRUCTURE
}

\author{
NGUYEN TIEN KHIEM \\ Institute of Mechanics
}

\begin{abstract}
The practical exploitation of engineering structures needs very much a simple criterion for checking safety of structures instead of reliability index that is very difficult to be computed within the probabilistic theory. This paper deals with establishment of a simple criterion for checking safety of column-like structure based on deterministic analysis. The criterion consists of bound of the flexural displacement at the top of column that is conducted from the displacement analysis combined with ultimate state for the structure.
\end{abstract}

\section{Statement of problem}

Checking safety of operating structures is an important problem in structural engineering. There are various safety criterias based on different concepts of limit states of structure. That is the ultimate, serviceability or fatigue limit structural states. The present investigation is concerned with the serviceability safety of structure and aimed to determine maximal allowable deflection of a column-like structure under flexural as well as axial load.

The commonly used criterion for checking the safety is based on comparison of load effect denoted by $Q$ with the resistance or strength of material $R$. The safety criterion in deterministic analysis therefore takes the form

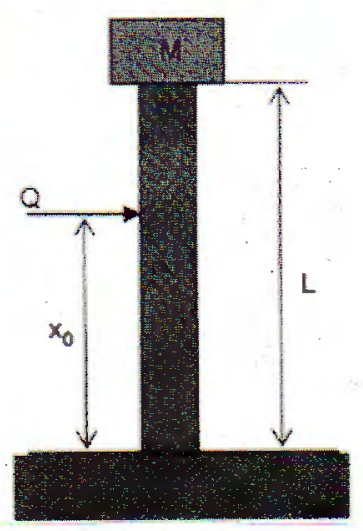

Fig. 1 .

$$
Q \prec R
$$

and limit state is represented by following equation

$$
R-Q=0 .
$$

The load effect $Q$ in the case of safety is represented by deflection $w$ of structure under a load $\mathrm{P}$ and the equation (1.2) results in maximal allowable deflection $w^{*}$ that due to equation (1.1) leads to the safety criterion

$$
w \leq w^{*} .
$$

In this study a vertical column consists of a beam with a concentrated mass $M$ at upper end and clamped at the lower one as shown in Fig. 1 is considered. It's, furthermore, 
assumed that the beam is subjected to a flexural distributed load with density $q(x)$, so that equation for flexural deflection $(w(x))$ has the form

$$
\frac{\partial^{2}}{\partial x^{2}}\left[E I \frac{\partial^{2} w}{\partial x^{2}}\right]+\frac{\partial}{\partial x}\left[P \frac{\partial w}{\partial x}\right]=q(x)
$$

where $P=M g$, or

$$
\frac{\partial^{2}}{\partial x^{2}}\left[E I \frac{\partial^{2} w}{\partial x^{2}}+P w\right]=q(x)
$$

Letting $f(x)$ be a function such that $\frac{\partial^{2} f}{\partial x^{2}}=q(x)$, one will get the equation

$$
\frac{\partial^{2}}{\partial x^{2}}\left[E I \frac{\partial^{2} w}{\partial x^{2}}+P w-f(x)\right]=0
$$

which consequently yields the equation

$$
E I \frac{\partial^{2} w}{\partial x^{2}}+P(w-C x-D)=f(x) .
$$

Introducing the notations

$$
u=w-C x-D, \quad \lambda^{2}=\frac{P}{E I},
$$

we obtain the final equation

$$
u^{\prime \prime}(x)+\lambda^{2} u(x)=\frac{1}{E I} f(x) .
$$

General solution of the later equation (1.5) has the form

$$
u(x)=A \cos \lambda x+B \sin \lambda x+(1 / \lambda E I) \int_{0}^{x} f(s) \sin \lambda(x-s) d s,
$$

so that

$$
w(x)=A \cos \lambda x+B \sin \lambda x+C x+D+(1 / \lambda E I) \int_{0}^{x} f(s) \sin \lambda(x-s) d s .
$$

Constants $A, B, C, D$ are determined using boundary conditions at both ends of the beam.

\section{Buckling condition}

Firstly, one will consider the case when $q(x)=0$ with the boundary condition of ideal cantilever beam

$$
w(0)=w^{\prime}(0)=w^{\prime \prime}(L)=w^{\prime \prime \prime}(L)+\lambda^{2} w^{\prime}(L)=0,
$$


that in combination with (1.6) yields the system of equations

$$
\begin{aligned}
& A+D=0 ; \quad B \lambda+C=0 ; \quad A \cos \lambda L+B \sin \lambda L=0 ; \\
& \lambda^{3} A \sin \lambda L-\lambda^{3} B \cos \lambda L-\lambda^{3} A \sin \lambda L+\lambda^{3} B \cos \lambda L-\lambda^{2} C=0 .
\end{aligned}
$$

From the algebraic equation it gives immediately $B=C=0$ and $A \cos \lambda L=0$. The last equation has nontrivial solution if

$$
\cos \lambda L=0
$$

or $\lambda L=(n-0.5) \pi, n=1,2,3, \ldots$ and in consequence

$$
P=P_{n}=\frac{(n-0.5)^{2} \pi^{2} E I}{L^{2}} .
$$

So that, stability condition for the beam is

$$
P=M g \prec P_{1}=\left(\frac{\pi}{2 L}\right)^{2} E I .
$$

Now we are going to consider the beam with flexible connection at the lower beam end $x=0$. Suppose that the connection may be treated as a rotational spring of the stiffness $\mathrm{K}$ such that boundary condition for the beam can be written as

$$
w(0)=E I w^{\prime \prime}(0)-K w^{\prime}(0)=w^{\prime \prime}(L)=w^{\prime \prime \prime}(L)+\lambda^{2} w^{\prime}(L)=0 .
$$

Substituting (1.6) into (2.5) leads to the equations

$$
\begin{aligned}
& A+D=0 ; \quad-\lambda^{2} E I \cdot A-K \cdot(B \lambda+C)=0 ; \quad-\lambda^{2} A \cos \lambda L-\lambda^{2} B \sin \lambda L=0 ; \\
& \lambda^{3} A \sin \lambda L-\lambda^{3} B \cos \lambda L-\lambda^{3} A \sin \lambda L+\lambda^{3} B \cos \lambda L-\lambda^{2} C=0,
\end{aligned}
$$

from which one gets $C=0$ and

$$
A \cos \lambda L+B \sin \lambda L=0 ; \lambda E I \cdot A+K \cdot B=0 .
$$

Condition for existence of nontrivial solution has the form

$$
K \cos \lambda L-\lambda E I \sin \lambda L=0
$$

or

$$
\beta \operatorname{tg} \beta=k,
$$

with the notations

$$
\beta=\lambda L ; \quad k=\frac{1}{\mu}=\frac{K L}{E I} .
$$

Suppose that solutions of equation (2.6) are $\beta_{j}=\beta_{j}(k), j=1,2,3, \ldots$, it is easy to verify that

$$
\beta_{j}(0)=(j-1) \pi \quad, \quad \beta_{j}(\infty)=(j-0.5) \pi
$$

and 


$$
\beta_{j}(0) \leq \beta_{j}(k) \leq \beta_{j}(\infty), j=1,2,3, \ldots
$$

So, the critical value of compressing load is

$$
P=P_{n}=\beta_{n}^{2}(k) \frac{E I}{L^{2}}
$$

and the stability condition takes the form

$$
P \prec\left(\frac{\beta_{1}(k)}{L}\right)^{2} E I \prec\left(\frac{\pi}{2 L}\right)^{2} E I .
$$

The inequality (2.9) implies that the column with flexible clamp is less stable than the cantilever one.

\section{Safety criterion in displacement}

In general case, i.e. when $q(x) \neq 0$, deflection curve has the form

$$
\begin{aligned}
& w(x)=A \cos \lambda x+B \sin \lambda x+C x+D+\frac{1}{\lambda E I} \int_{0}^{x} f(s) \sin \lambda(x-s) d s \\
& w^{\prime}(x)=-\lambda A \sin \lambda x+\lambda B \cos \lambda x+C+\frac{1}{E I} \int_{0}^{x} f(s) \cos \lambda(x-s) d s \\
& w^{\prime \prime}(x)=-\lambda^{2} A \cos \lambda x-\lambda^{2} B \sin \lambda x-\frac{\lambda}{E I} \int_{0}^{x} f(s) \sin \lambda(x-s) d s+\frac{f(x)}{E I} \\
& w^{\prime \prime \prime}(x)=\lambda^{3} A \sin \lambda x-\lambda^{3} B \cos \lambda x-\frac{\lambda^{2}}{E I} \int_{0}^{x} f(s) \cos \lambda(x-s) d s+\frac{f^{\prime}(x)}{E I}
\end{aligned}
$$

By analogy to the above, we distinguish two cases, rigid and flexible clamp of column and let's go to consider both them.

\subsection{Cantilever beam}

In this case, the boundary conditions are

and therefore one obtains

$$
w(0)=w^{\prime}(0)=w^{\prime \prime}(L)=w^{\prime \prime \prime}(L)+\lambda^{2} w^{\prime}(L)=0
$$

$$
\begin{aligned}
& A+D=0 ; \quad B \lambda+C=0 ; \quad \lambda^{2} C+\frac{f^{\prime}(L)}{E I}=0 ; \\
& A \cos \lambda L+B \sin \lambda L=\frac{f(L)}{\lambda^{2}} E I-\frac{\lambda^{2}}{E I} \int_{0}^{L} f(s) \sin \lambda(L-s) d s .
\end{aligned}
$$


Let the function $f(x)$ be chosen so that it satisfies equations

$$
f(L)=f^{\prime}(L)=0,
$$

which results in $B=C=0$ and

$$
A \cos \lambda L=-\frac{1}{\lambda E I} \int_{0}^{L} f(s) \sin \lambda(L-s) d s .
$$

The last equation has nontrivial solution if

then

$$
\cos \lambda L \neq 0,
$$

$$
A=-\frac{1}{\lambda E I \cos \lambda L} \int_{0}^{L} f(s) \sin \lambda(L-s) d s .
$$

Substituting the obtained constants $A, B, C, D$ into (3.1) one will have

$$
w(x)=\frac{1-\cos \lambda x}{\lambda E I \cos \lambda L} \int_{0}^{L} f(s) \sin \lambda(L-s) d s+\frac{1}{\lambda E I} \int_{0}^{x} f(s) \sin \lambda(x-s) d s .
$$

Using equation (3.4), the deflection at the column top can be calculated as

$$
w(L)=\frac{1}{\lambda E I \cos \lambda L} \int_{0}^{L} f(s) \sin \lambda(L-s) d s .
$$

Furthermore, letting $\lambda \rightarrow 0$ (i. e. there is no compressing load $P=0$ ), one obtains

$$
\begin{aligned}
\lim _{\lambda \rightarrow 0} w(L) & =\frac{1}{E I} \int_{0}^{L} f(s)(L-s) \lim _{\lambda \rightarrow 0} \frac{\sin \lambda(L-s)}{\lambda(L-s) \cos \lambda L} d s \\
& =\frac{1}{E I} \int_{0}^{L} f(s)(L-s) \cdot d s
\end{aligned}
$$

\subsection{Column with flexible connection}

Equations (3.1) with the boundary conditions (2.5) yields the system of equations

$$
\begin{aligned}
A+D=0 ; \quad C & =0 ; \quad \lambda^{2} E I \cdot A+\lambda K \cdot B=f(0) . \\
A \cos \lambda L+B \sin \lambda L & =-(1 / \lambda E I) \int_{0}^{L} f(s) \sin \lambda(L-s) d s .
\end{aligned}
$$

Letting $\Delta=\lambda^{2} E I \sin \lambda L-\lambda K \cos \lambda L$, one will have 


$$
\begin{gathered}
\left.A=(1 / \Delta)\{f(0) \sin \lambda L+(K / E I)) \int_{0}^{L} f(s) \sin \lambda(L-s) d s\right\} ; \\
B=(-1 / \Delta)\left\{f(0) \cos \lambda L+\lambda \int_{0}^{L} f(s) \sin \lambda(L-s) d s\right\} .
\end{gathered}
$$

Finally, the deflection curve and deflection at the column top are respectively

$$
\begin{aligned}
& w(x)=A(\cos \lambda x-1)+B \sin \lambda x+\frac{1}{\lambda E I} \int_{0}^{x} f(s) \sin \lambda(x-s) d s, \\
& w(L)=A(\cos \lambda L-1)+B \sin \lambda L+\frac{1}{\lambda E I} \int_{0}^{L} f(s) \sin \lambda(L-s) d s .
\end{aligned}
$$

Under $\lambda$ approaching to zero one has got

$$
\lim _{\lambda \rightarrow 0} w(L)=(1 / K)\left[f(0) L+(1 / \lambda E I) \int_{0}^{L} f(s)(L-s) d s\right] .
$$

\subsection{The case of concentrated flexural loading}

Let's consider the case when $q(x)=Q_{0} \delta\left(x-x_{0}\right)$, then we will have a boundary problem

$$
f^{\prime \prime}(x)=Q_{0} \delta\left(x-x_{0}\right), \quad f(L)=f^{\prime}(L)=0 .
$$

the general solution of which is

$$
f(x)=C x+D+\left\{\begin{array}{l}
0 \quad \text { for } x \leq x_{0} \\
Q_{0}\left(x-x_{0}\right) \text { for } x \geq x_{0}
\end{array} .\right.
$$

Satisfying the boundary conditions leads to

$$
D=f(0)=Q_{0} x_{0}, C=f^{\prime}(0)=-Q_{0}
$$

thus

$$
f(x)=-Q_{0} x+Q_{0} x_{0}+\left\{\begin{array}{l}
0 \quad \text { for } x \leq x_{0} \\
Q_{0}\left(x-x_{0}\right) \text { for } x \geq x_{0}
\end{array}=\left\{\begin{array}{l}
Q_{0}\left(x_{0}-x\right) \text { for } x \leq x_{0} \\
0 \quad \text { for } x \geq x_{0}
\end{array} .\right.\right.
$$

Using this function $f(x)$ one can calculate the integral

$$
\begin{gathered}
J=\int_{0}^{L} f(s) \sin \lambda(L-s) d s=Q_{0} \int_{0}^{x_{0}}\left(x_{0}-s\right) \sin \lambda(L-s) d s= \\
=Q_{0} L^{2} \int_{0}^{\bar{x}_{0}}\left(\bar{x}_{0}-s\right) \sin \beta(1-s) d s=Q_{0} L^{2} G\left(\bar{x}_{0}, \beta\right) ;
\end{gathered}
$$


where $\beta=\lambda L ; \quad \bar{x}_{0}=x_{0} / L$ and

$$
G\left(\bar{x}_{0}, \beta\right)=-\frac{1}{\beta^{2}}\left[\sin \beta\left(1-\bar{x}_{0}\right)+\beta \bar{x}_{0} \cos \beta-\sin \beta\right] .
$$

Furthermore, it can be calculated the limit

$$
\lim _{\beta \rightarrow 0} G\left(\bar{x}_{0}, \beta\right)=0 ; \lim _{\beta \rightarrow 0} \frac{G\left(\bar{x}_{0}, \beta\right)}{\beta}=\frac{\bar{x}_{0}^{2}\left(3-\bar{x}_{0}\right)}{6} .
$$

Therefore, deflection at the top of column in rigid and flexible connection respectively will take the form

$$
w_{1}(L)=\frac{1}{\lambda E I \cos \lambda L} \int_{0}^{L} f(s) \sin \lambda(L-s) d s=\frac{Q_{0} L^{3} G\left(\bar{x}_{0}, \beta\right)}{E I \beta \cos \beta}
$$

and

$$
w_{2}(L)=\frac{Q_{0} L^{3}}{E I \beta} \frac{\bar{x}_{0} \mu \sin \beta+G}{\cos \beta-\beta \mu \sin \beta},
$$

after the constants have been calculated as

$$
\begin{gathered}
A=\frac{Q_{0} L^{3}}{E I \beta} \frac{\bar{x}_{0} \sin \beta+k G}{\beta \sin \beta-k \cos \beta} ; B=-\frac{Q_{0} L^{3}}{E I \beta} \frac{\bar{x}_{0} \cos \beta+\beta G}{\beta \sin \beta-k \cos \beta}, \\
\mu=1 / k=E I / K L(=0 \text { as } K \rightarrow \infty) .
\end{gathered}
$$

Approaching $\lambda$ to zero, one could obtain the deflections at the column top as

$$
\begin{aligned}
& \lim _{\lambda \rightarrow 0} w_{1}(L)=\frac{Q_{0} L^{3}}{6 E I} \bar{x}_{0}^{2}\left(3-\bar{x}_{0}\right)=w_{10}(L) \\
& \lim _{\lambda \rightarrow 0} w_{2}(L)=\frac{Q_{0} L^{3} \bar{x}_{0}}{6 E I}\left[6 \mu+\bar{x}_{0}\left(3-\bar{x}_{0}\right)\right]=w_{20}(L) .
\end{aligned}
$$

\subsection{Safety criterion or maximal deflection of the column top}

It is well known that the stress of a cantilever beam used in checking its load-carrying capacity is

$$
\sigma=\frac{M D}{2 I}
$$

where $M$ - bending moment at the lowest section, $D$ characteristic diameter of the cross section and $I$ is sectional moment of inertia. Let the stress limit for the material be denoted by $[\sigma]$, so that it must be satisfied the condition

$$
\sigma=\frac{M D}{2 I} \leq[\sigma]
$$

From the condition (3.17) it's seen that for checking the beam load-carrying capacity the bending moment at lower end of column 


$$
M=E I w^{\prime \prime}(0)=-\lambda^{2} E I \cdot A+f(0) .
$$

should be calculated. In the case of rigidly cantidessered column one has

$$
M=f(0)+\frac{\lambda}{\cos \lambda L} \int_{0}^{L} f(s) \sin \lambda(L-s) d s .
$$

For the concentrated load it takes the form

$$
\begin{aligned}
M & =Q_{0} x_{0}+Q_{0} L^{2} \lambda G / \cos \beta=Q_{0} L\left[\bar{x}_{0}+\beta G / \cos \beta\right]= \\
& =Q_{0} L\left[\left(\sin \beta-\sin \beta\left(1-\bar{x}_{0}\right)\right) / \beta \cos \beta\right]
\end{aligned}
$$

If there is no axial loading, the moment would be

$$
\lim _{\beta \rightarrow 0} M=Q_{0} L \bar{x}_{0}=Q_{0} x_{0}
$$

and the condition (3.17) gives

$$
\frac{3 w_{10} x_{0} D E}{L^{3} \bar{x}_{0}^{2}\left(3-\bar{x}_{0}\right)} \leq[\sigma]
$$

or

$$
\bar{w}_{10}=\frac{w_{10}}{L} \leq\left[\bar{w}_{10}\right]=\frac{[\sigma]}{E \gamma} \frac{\bar{x}_{0}\left(3-\bar{x}_{0}\right)}{3},
$$

where $\gamma=D / L$ representing the thinness of the beam. The function $x(1-x / 3)$ in the interval $[0,1]$ reaches maximum value at $\mathrm{x}=1$ and equals $2 / 3$, so that the maximal allowable deflection at the column top in this case is

$$
\left[\bar{w}_{10}\right]=\frac{2[\sigma]}{3 E \gamma}=\frac{2 L}{3 D} \frac{[\sigma]}{E} .
$$

For steel, when $\frac{[\sigma]}{E}=0.1 \%$ the limit deflection could be much more simplified as

$$
\left[\bar{w}_{10}\right]=\frac{L}{15 D}(\%) .
$$

In the case of presence of axial load condition (3.17) takes the form

$$
\sigma=\frac{w_{1}}{L} \frac{D}{L} \frac{E}{2} \frac{\beta \cos \beta}{G}\left[\frac{\sin \beta-\sin \beta\left(1-\bar{x}_{0}\right)}{\beta \cos \beta}\right] \leq[\sigma] .
$$

Furthermore, the limit deflection can be determined as follows

$$
\bar{w}_{1}=\frac{w_{1}}{L} \leq\left[\bar{w}_{1}\right]=\frac{[\sigma]}{E \gamma} H_{1}\left(\beta, \bar{x}_{0}\right),
$$

where $H_{1}\left(\beta, \bar{x}_{0}\right)=\frac{2\left[\beta \bar{x}_{0} \cos \beta+\sin \beta\left(1-\bar{x}_{0}\right)-\sin \beta\right]}{\beta^{2}\left[\sin \beta\left(1-\bar{x}_{0}\right)-\sin \beta\right]}$.

It's easy to get 


$$
\lim _{\beta \rightarrow 0} H_{1}\left(\beta, \bar{x}_{0}\right)=\bar{x}_{0} \frac{3-\bar{x}_{0}}{3},
$$

which shows that the equation (3.18) is a particularity of the equation (3.20).

In the case of flexibly cantilevered column, using the expressions of the constant $A$ and deflection at the column top, the moment can be calculated as

$$
\begin{aligned}
M & =Q_{0} x_{0}+Q_{0} L \frac{\beta\left(G+\bar{x}_{0} \mu \sin \beta\right)}{\cos \beta-\beta \mu \sin \beta}=Q_{0} L\left[\bar{x}_{0}+\frac{\beta\left(G+\bar{x}_{0} \mu \sin \beta\right)}{\cos \beta-\beta \mu \sin \beta}\right]= \\
& =Q_{0} L\left[\frac{\sin \beta-\sin \beta\left(1-\bar{x}_{0}\right)}{\beta(\cos \beta-\beta \mu \sin \beta)}\right] .
\end{aligned}
$$

So that the condition (3.17) leads to the equation

$$
\bar{w}_{2}=\frac{w_{2}}{L} \leq\left[\bar{w}_{2}\right]=\frac{[\sigma]}{E \gamma} H\left(\beta, \mu, \bar{x}_{0}\right),
$$

where

$$
H\left(\beta, \mu, \bar{x}_{0}\right)=\frac{2\left[\beta \bar{x}_{0}(\cos \beta-\beta \mu \sin \beta)+\sin \beta\left(1-\bar{x}_{0}\right)-\sin \beta\right]}{\beta^{2}\left[\sin \beta\left(1-\bar{x}_{0}\right)-\sin \beta\right]} .
$$

The later function has the limit $\lim _{\beta \rightarrow 0} H\left(\beta, \bar{x}_{0}\right)=2 \mu+\bar{x}_{0}\left(3-\bar{x}_{0}\right) / 3$, so that maximal allowable deflection at the top of the flexibly cantilevered column without axial load takes the form

$$
\left[\bar{w}_{20}\right]=\frac{[\sigma]}{E \gamma}\left[2 \mu+\frac{\bar{x}_{0}\left(3-\bar{x}_{0}\right)}{3}\right] .
$$

The formula (3.23) shows that flexibility of the connection increases the safety margin of the column in deflection and if $\mu=0$ the equation becomes (3.18).

The effect of axial load, position of flexural load application and flexibility of connection on the safety margin of the column can be studied by consideration of graphics of the function (3.22) with respect to parameters $\beta, \bar{x}_{0}, \mu$. The Fig. 2 shows the graphics of the function $\mathrm{H}$ versus $\beta$ with different values of parameters $\bar{x}_{0}, \mu$. Namely, four pictures given in Fig. 2 correspond to four values of $\mu=0 ; 0.1 ; 0.3 ; 0.5$ and in each picture there are 10 curves correspondingly to 10 values of $\bar{x}_{0}=0.1-1.0$. It is clear that the coefficient $\mathrm{H}$ increases with increasing of $\mu$, that implies enlargement of safety margin when connection becomes more flexible. It is also seen that the higher coefficient $\mathrm{H}$ is the closer position of load application to column top will be. The higher point flexual load application is, the more safety margin of the beam in top displacement. In each pictures mentioned above one can recognize that all curves with different values of $\bar{x}_{0}$ intersect at a point corresponding to $\beta$ which is the solution of the equation (2.6) or $\beta \operatorname{tg} \beta=1 / \mu$. From the first picture in Fig. 2 corresponding to $\mu=0$ (rigid connection or ideal clamp) it's seen that the intersection point is $\beta=\pi / 2$. 

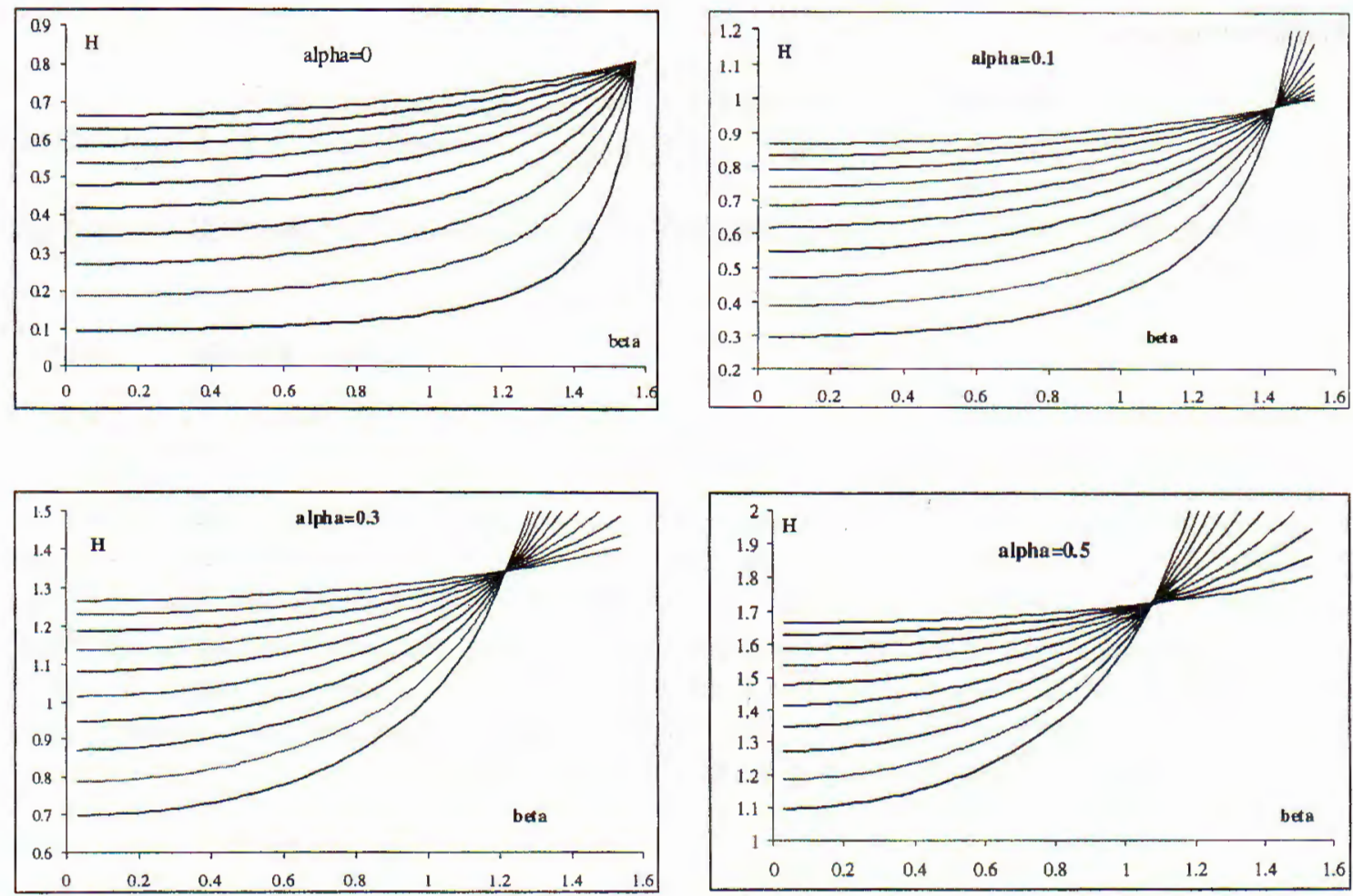

Fig. 2

\section{Conclusion}

Thus, in this paper the results have been newly presented as follow:

- The stability condition for column with flexible connection with base has been conducted by the well known classical method. The obtained criterion shows that the critical load in case of flexible connection becomes less than that of rigid connection, i. e. flexible connection with base makes the column less stable.

- The safety criterion with respect to maximal deflection of column under both the flexural and axial loads was obtained in general case of boundary connection and loading. The result shows that the maximal deflection is reached when flexural is applied to the top of column and flexibility of connection at the base enlarges the safety margin (limit deflection at the top).

- The obtained results can be applied instantly to checking the safety of structures operating in Vietnam shelf.

Acknowledgement. This work has been completed under support of the National Council in natural sciences of Vietnam, to whom the author is sincerely thankful. 


\section{References}

1. Volmir A. S., Stability of Deformable Systems, Nauka, Moscow, 1967.

2. Madsen H. O., Krenk S. and Lind N. C., Methods of Structural Safety, Printice-Hall, Inc. New Jersey, 1986.

3. Nowak A. S. and Collins K. R., Reliability of Structures, McGraw-Hill, Singapore, 2000 .

Received October 5, 2004

\section{VỀ MỘT ĐIỀU KIỆN AN TOANN KẾT CẤU CÔNG TRÌNH DẠNG CộT}

Hiện nay trong thực tế khai thác sử dụng các công trình, dặc biệt là công trình trên biển rất cần một tiêu chuẩn an toàn đơn giản và dễ áp dụng, không phải là điều kiện an toàn phức tạp như trong lý thuyết độ tin cậy quy định. Bài báo này có mục đích đưa ra một điều kiện đơn giản để kiểm tra đánh giá độ an toàn của công trình dạng cột đứng dưới tác động của tải trọng ngang và dọc trục. Điều kiện an toàn này được biểu diễn bằng chuyển vị lớn nhất có thể của đỉnh cột được xây dựng dựa trên lời giải bài toán chuyển vị kết hợp với điều kiện tới hạn bền thông thường. 